\title{
versants
}

\section{«With a slip of the pen you». Amelia Rosselli e Boris Pasternak}

\author{
Sara SERMini \\ Università della Svizzera italiana
}

\begin{abstract}
Partendo dall'articolo su Boris Pasternak scritto da Amelia Rosselli nel I966 e attraverso l'indagine delle edizioni del poeta russo conservate presso la biblioteca della poetessa, mi propongo in questo breve scritto di studiare i motivi letterari e politici della vicinanza di Amelia Rosselli all'opera di Boris Pasternak.

Keywords: Amelia Rosselli, Boris Pasternak, «Avanti», Documento, umanesimo
\end{abstract}

Sul supplemento domenicale dell'«Avanti» del 4 dicembre 1966 escono dieci poesie di Boris Pasternak scelte da Amelia Rosselli e accompagnate da un suo breve scritto dal titolo La fatica di essere autentico.' Oltre ad inaugurare la collaborazione di Rosselli con alcuni quotidiani italiani, ${ }^{2}$ l'articolo costituisce anche il suo primo intervento in assoluto su uno scrittore e sulla sua opera letteraria.

Come ha messo in luce Francesca Caputo, Rosselli riferisce al fratello John del dissenso di Emilio Lussu ${ }^{3}$ circa la decisione di lavorare per un quotidiano come l'«Avanti», considerato «di destra», e commenta: «but I personally think that as long as I don't write on fascist papers, literary subjects can be affrontati on any paper: especially as I tend to give them left-wing substance». ${ }^{4}$ Sebbene l'articolo sul poeta russo apparentemente non presenti caratteri di militanza politica, vale la pena di provare a dar peso a questa affermazione per capire a fondo i motivi della vicinanza di Amelia Rosselli all'opera di Pasternak, il quale è riconosciuto a più riprese dalla poetessa

I Dieci pagine di Pasternak scelte da Rosselli, a cura di Walter Pedullà, in «Avanti», 4 dicembre I966 (comprende Amelia Rosselli, La fatica di essere autentico; ora in Ead., Una scrittura plurale. Saggi e interventi critici, a cura di Francesca Caputo, Novara, Interlinea, 2004). Le poesie di Pasternak pubblicate sono le seguenti: Febbraio [1912]; Tre varianti [1925]; Marburgo [1915].

2 Cfr. Francesca Caputo, «Cercare la parola che esprima gli altri», prefazione a Rosselli, Una scrittura plurale. Saggi e interventi critici, cit., p. I2: «Fra il 1967 e il 1970 si alterneranno sporadiche collaborazioni al quotidiano socialista, a "Paese sera", all' "Unità"; nel decennio successivo fra il 1975 e il 1976 scriverà alcuni pezzi per "La Stampa" (a cui era stata segnalata da Alberto Moravia) e ancora, dal 1978 al 1979, per "Paese Sera". Suoi scritti compariranno inoltre, in modo saltuario, su altri periodici (da "Rinascita" a "Nuovi Argomenti", da "La Fiera Letterata" a "Poesia")».

3 Emilio Lussu è intimamente legato alla famiglia Rosselli per la sua intensa attività all'interno di Giustizia e Libertà. I Lussu saranno un punto di riferimento fondamentale per Amelia Rosselli dopo il suo trasferimento a Roma nel 1949.

4 Rosselli, Una scrittura plurale. Saggi e interventi critici, cit., nota I5, p. 22. 
come una delle maggiori fonti di ispirazione per i suoi versi e in particolare per la raccolta Documento, iniziata proprio nel $1966 .{ }^{5}$

Dei dieci componimenti scelti soltanto tre sono classificabili sotto l'etichetta di "poesie "politiche"»" poeta si sforza «d'essere più ragionevole, meno sorpreso, partecipe ai fatti come se veramente gli fossero vicini»7: due componimenti tratti dal poema Il luogotenente Schmidt (nella traduzione "splendida» ${ }^{8}$ di Angelo Maria Ripellino) e un estratto dal poema Spektorskij nella traduzione di Pietro Zveteremich uscita per l'appunto in quell'anno. ${ }^{9}$ I due poemi, pubblicati rispettivamente nel I926 e nel 193 I $^{10}{ }^{10}$ sono imperniati su due figure politicamente rilevanti pur nella loro sostanziale diversità: Pëtr Petrović Schmidt, luogotenente della flotta del Mar Nero a capo dell'insurrezione di Sebastopoli del I905, ${ }^{\text {II }}$ eletto a simbolo dell'«intellettuale che s'immola per la rivoluzione, dibattendosi sino all'estremo fra l'indecisione, l'ansia delle riforme, l'orrore della strage», ${ }^{12}$ e Sergeij Spektorskij, giovane intellettuale nella Mosca

5 Sulla genesi della raccolta cfr. Amelia Rosselli, L'opera poetica, a cura di Stefano Giovannuzzi e con un saggio introduttivo di Emmanuela Tandello, apparati critici di Francesco Carbognin, Chiara Carpita, Silvia De March, Stefano Giovannuzzi, Gabriella Palli Baroni, Emmanuella Tandello, Milano, Mondadori, I Meridiani, 2013.

6 Rosselli, La fatica di essere autentico, cit., p. $\mathbf{2 2 9 .}$

7 Ibidem.

8 Ibidem. Così Rosselli definisce la traduzione di Angelo Maria Ripellino delle Poesie che Rosselli possiede nella ristampa del 1962 (Boris Pasternak, Poesie, trad. di Angelo Maria Ripellino, Torino, Einaudi, 1962) conservata presso il Fondo Amelia Rosselli della Biblioteca della Facoltà di Lingue dell'Università della Tuscia di Viterbo (FAR 2435). D'ora in avanti, i libri presenti presso il Fondo Amelia Rosselli di Viterbo saranno contraddistinti dalla sigla FAR, seguita dal numero della segnatura (secondo la catalogazione disponibile online sul sito: www.biblioteche.unitus.it).

9 Boris Pasternak, Poesie inedite, con un saggio di Andrej Sinjavskij, trad. di Pietro Zveteremich con la collaborazione di Domenico Porzio, Milano, Rizzoli, 1966, FAR 217.

Io La genesi del poema Spektorskij va dal 1924 al I930; esso fu tuttavia pubblicato integralmente soltanto nel 1965. Scrive Christopher Barnes a proposito del poema: «Concepito come una lunga prosa poetica, o novella i versi, provocò l'immediata comparazione con l'Eugenio Onegin. Pushkin aveva usato il tetrametro giambico; Pasternak usò quartine di pentametri giambici» (Christopher Barnes, Boris Pasternak, I89o-1928. A Literary Biography, Cambridge University Press, 1989, vol. I, p. 350. La traduzione è mia).

II Per quanto riguarda la scelta del soggetto Simon Karlinsky, nella sua biografia su Marina Cvetaeva, racconta di come Pasternak, ispirato dai due poemi della Cvetaeva (L'accalappiatopi e il Poema della fine), decise di «cimentarsi nel poema narrativo, un genere in cui non s'era mai provato prima d'allora. Cercò un soggetto e lo trovò nelle risposte della Cvetaeva al questionario per il progettato dizionario bibliografico degli scrittori del Novecento che lui stesso le aveva inviato nell'aprile di quell'anno. La Cvetaeva vi ribadiva l'infatuazione per la rivoluzione del 1905 e per il suo martire, il luogotenente Pëtr Petrović Schmidt, oltre all'avversione per le rivoluzioni del febbraio e dell'ottobre del I9I7. In primavera, Pasternak, prese a lavorare all'Anno 1905 e al Luogotenente Schmidt...» (Simon Karlinsky, Marina Cvetaeva, Napoli, Guida editori, 1989, p. 197). Sulla rivoluzione del $1905 \mathrm{cfr}$. Orlando Figes, La tragedia di un popolo. La Rivoluzione russa I89I-I924, trad. di Raffaelle Petrillo, Milano, Corbaccio, 1997.

I2 Cito da Angelo Maria Ripellino, introduzione a Boris Pasternak [1957], Poesie, Torino, Ei- 
pre-rivoluzionaria attraverso la cui figura Pasternak tenta la via di un impegno ideologico.

Dal primo poema, in particolare, Rosselli estrapola quei passi in grado di mostrare come Pasternak «innamorato anche della rivoluzione non volle glorificarsi decantandola, ma solo nominare un uomo, e in ogni sua precisione descrivercelo come un uomo qualunque». ${ }^{13}$ Il luogotenente Schmidt scelto da Rosselli è un uomo che ricorda i fatti dei quali è stato protagonista come se fossero capitati per caso e poi trascorsi nell'inesorabile fluire dei giorni. Nessun accento rivoluzionario, piuttosto uno sguardo introspettivo pieno di stupore:

«Io ho l'ardire di scrivervi. Sarà necessario ravvivare il ricordo? Sono il marinaio del derby.

Allora mi proponeste un enigma.

D’altronde più tardi, più tardi. C'è tempo.

Quando vi scorsi... Ma sino a quel giorno vivevo alla meglio, e d'un tratto me ne scordai, e mi misi d'un colpo ad incalzarvi con lo sguardo e vi perdetti tra la folla dietro a un arganello.

Quando cessò il torpore della mia sconvenienza, mi accorsi di non sapere chi foste.

Il séguito è noto. È difficile intendersi, per incontrarsi in maniera così favolosa di nuovo.

Avete almeno meditato quanta ampiezza ci sia qui per la fede? - Crucciarsi d'uno sguardo, sparire tra la folla, capitare di notte in un treno, raggiustare l'ombrello e ritrovarsi accanto!» ${ }^{14}$

Pasternak tratteggia con poche pennellate la vicenda rivoluzionaria e la abbandona sullo sfondo per «riportare solo il dato, il fatto, il carattere dell'uomo $»^{15}$ e i suoi sentimenti, come nella seconda poesia scelta da Rosselli in cui l'indefinito $t u$, al quale «il marinaio del derby» si rivolge a cuore aperto, diventa oggetto di un amore rimasto incompiuto per destino. Anche l'orrore della strage rimane eco appena udibile di una notizia ormai di seconda mano:

naudi, 2009, p. XXV.

I3 Rosselli, La fatica di essere autentico, cit., p. I29.

I4 Cito da Boris Pasternak, Poesie, a cura di Angelo Maria Ripellino, Torino, Einaudi, 2009, p. 315. Le virgolette sono dell'autore.

I5 Rosselli, La fatica di essere autentico, cit., pp. I29-I30. 
[...]

«Dov'è il giorno in cui, aperto un telegramma, tutto dimenticando al tuo "per sempre", già sognavo di accorrere e arrivare all'improvviso?

Ma come, tu dirai, son capitato qui?

La sera in cui giunse c'era un comizio. Io presagivo il naufragio della rivolta, ma nulla ormai poteva persuaderli. Quella notte partire significava svignarsela.

Oh come ti bramavo! Fu un supplizio attaccare sapendo che il popolo non era pronto, sacrificare il convegno e vedere in eccesso argomenti a favore delle altre città.

La fede nel viaggio per zone industriali, in un nuovo sciopero e in un nuovo slancio s'intrecciava in me, forse, col segreto sentore che saremmo andati insieme.

Ma affluì l'ondata delle deputazioni, la Duma, i socialisti, uno squillo dietro l'altro. Non si poteva nemmeno tentar di partire. Ecco, e così son finito ribelle.

Questo è tutto, mi pare. Sono assai più tranquillo di quanto essi s'aspettino. Che cosa ancora, dunque? Già, riguardo al massacro di Sebastopoli c'è nei vecchi giornali un completo resoconto.» ${ }^{16}$

Qualsiasi forma di eroismo perde di valore nell'opera di Pasternak mentre la rivolta, scrive Rosselli, diventa «il segno di una normalità non necessariamente eroica; spesso atto di natura, o semplice onestà, necessità». ${ }^{17} \mathrm{Ri}$ badisce il poeta in un passo di Spektorskij, fatto ristampare da Rosselli sulle pagine dell'«Avanti»:

Per l'eroe non avrei dato niente e non presto avrei preso a ragionare di lui, ma ciò di cui scrivevo era una scatola di raggi nella quale lui si profilava innanzi a me $[. . .]^{18}$

I6 Pasternak, Poesie, cit., pp. 373-375. Anche in questo caso le virgolette sono dell'autore.

I7 Rosselli, La fatica di essere autentico, cit., p. I29.

I8 Cfr. Dieci pagine di Pasternak scelte da Rosselli, cit., p. I. 
Come ha scritto Angelo Maria Ripellino, «la lirica di Pasternak è tessuta di elementari sensazioni psichiche». ${ }^{19}$ Pasternak si ferma ad osservare gli uomini per ritrarli nella quotidianità delle loro azioni, dei loro bisogni e dei loro sentimenti. Per dirla con un verso di Rosselli, al centro dell'opera di Pasternak c'è «l'uomo con le sue variegate variopinte pene $»^{20}$ e ciò risulta ancor più evidente nella prosa che nella poesia:

Sorprendente è poi il leggere la sua prosa (non solo lo Zivago, ma anche per esempio l'autobiografia Salvacondotto): molto più cauta si fa la sua poesia nella prosa, in una ricerca del "banale" come punto alto e d'obbligo della vita. Invece nei versi egli è tutto "inedito", con tematiche impensate, con un rovesciamento del banale per mostrarne i più patetici segreti. ${ }^{21}$

Per quanto riguarda lo stile della poesia di Pasternak, Rosselli è attratta dalla «sua calma», come ribadirà in un'intervista del I99I, dal «suo dire con apparente facilità cose assai complesse»:22

La calma di Pasternak era invincibile, nascosta, nascosto anche tutto quello che poteva essere il suo tormento, tormentosissimo lavorio, tormentosissima preparazione alla calma, al vaso non rotto, alla poesia fatta, decisa in partenza, senza pretese eppure in un balzo scombussolando tutti i program$\mathrm{mi}$, le poetiche pubbliche. ${ }^{23}$

La calma di Pasternak si rivela nei dettagli, «nelle minuzie più sottili», ${ }^{24}$ nel suo saper descrivere un tempo che lentamente, ineludibilmente trascorre e i cui attimi di sospensione respirano soltanto all'interno dei «lucidi quadri ${ }^{25}$ poetici. ${ }^{26}$ Ed è proprio questa calma ad essere in grado di combattere la massa di coloro che «agitandosi, inghiot tono gnocchi / di parole impensabili con desinenza in -ismo»; ${ }^{27}$ soltanto la calma sembra essere capace di mettere

I9 Ripellino, introduzione a Boris Pasternak, Poesie, cit., p. XIV.

20 Rosselli, Variazioni belliche, ora in Ead., L'opera poetica, cit., p. 29.

2I Rosselli, La fatica di essere autentico..., cit., p. I30.

22 Giovanni Salviati, Nel linguaggio della realtà. Conversazione con Amelia Rosselli [199I], in «clanDestino», X, I, gennaio-marzo I997, pp. 9-I3; ora in Rosselli, È vostra la vita che ho perso, a cura di Monica Venturini e Silvia De March, prefazione di Laura Barile, Firenze, Le Lettere, 20I0, p. 219.

23 Rosselli, La fatica di essere autentico, cit., p. 129.

24 Pasternak, Febbraio, in Id., Poesie, cit., p. 3. La poesia è riprodotta per volere di Amelia Rosselli sulle pagine dell'«Avanti».

25 Rosselli, La fatica di essere autentico, cit., p. 130.

26 A proposito del fluire del tempo, in una poesia dal titolo Dichiarazione, tratta dal Dottor Zivago e selezionata da Amelia Rosselli, si legge: «La vita è tornata, così, senza motivo / come allora che s'era stranamente interrotta».

27 Cfr. Pasternak, Il luogotenente Schmidt, in ivi, pp. 329. 
in crisi la piega totalitaria di una rivoluzione nella quale il poeta aveva inizialmente creduto. Anche Rosselli, nella raccolta Documento, compie un'operazione simile sulla scia del poeta russo:

Mille, piccoli oggetti delicati, la

falce e il martello sono divenuti punti

interrogativi.

La mia vita privata è un best-seller

e i miei romanzi d'amore sono luride facilitazioni

e io ne riconosco l'errore.

La neve scende piana svolazzando contenta

il mio cuore si rabbuia attentamente

si contrae e si perdona con uno schiaffo

fatto su misura. Oh il vento è leggero!

E non vuol disturbare l'ordine della

pace in terra. ${ }^{28}$

In un'intervista condotta da Guido Galeno e Orazio Converso nel I987, dopo aver letto la poesia Neve (in cui, similmente ai versi sopra citati, i fiocchi di neve sono descritti come «minuscoli oggetti festeggianti»), ${ }^{29}$ Rosselli afferma:

AR: Ma il libro era un pochino ispirato da Pasternak, dalla poesia di Pasternak.

GG: Con la neve...? C'è sempre la neve.

AR: Sì, ma un po' malinconico, pasternakiano [...] ha una tecnica formidabile, supera di molto i nostri più cattivi, cattivi in senso tecnico, più crudeli poeti. Ma nella media lui cerca una semplificazione, esprime i sentimenti di tutti, se può. Tra i due estremi. ${ }^{30}$

Dalle lettere al fratello John possiamo far risalire la prima lettura di Pasternak da parte di Rosselli intorno al 1964, quando Rosselli scrive: «reading Pasternak's poetry which is rather good though I find his novel somewhat dull».31 Se la maggior parte degli scrittori italiani in quegli anni

28 Rosselli, Documento, ora in Ead., L'opera poetica, cit., p. 408.

29 Ibidem, p. 407.

30 Intervista per numero I di «Videor» (videorivista diretta da Elio Pagliarani ed edita da Orazio Converso), ora trascitta in Rosselli, Ė vostra la vita che ho perso, cit., p. 219.

3I Da una lettera al fratello John Rosselli del Io agosto I964, conservata presso il Centro Manoscritti dell'Università di Pavia. 
avevano recensito Il dottor Zivago, Rosselli (che lo legge nel I964, a distanza di qualche anno dalla sua prima edizione) $)^{32}$ lo definisce privatamente come dull, "sbiadito", "noioso", mentre pubblicamente, nel suo articolo, si limita a liquidarlo in un breve accenno, dando attenzione solamente alle poesie raccolte nel Dottor Zivago. Oltre al noto romanzo e alle poesie di Pasternak, Rosselli conosce anche alcune traduzioni in lingua inglese dell'autore russo («tradotto in inglese poi Pasternak ha tutt'altra faccia: a volte ne fanno un accademico»). ${ }^{33}$ Sugli scaffali della sua biblioteca si scorge l'edizione di un breve racconto autobiografico dal titolo The last summer ${ }^{34}$ nonché Safe con$d u c t,{ }^{35}$ traduzione inglese dell'autobiografia di Pasternak.

Ma soffermiamoci brevemente sul racconto, edito per la prima volta nel I934 con il titolo Povest' (ossia Un racconto), il quale presenta una significativa consonanza con l'opera e il pensiero di Amelia Rosselli, sulla scia di quell'umanesimo, di quella attenzione all'uomo sin qui delineata.

Il senso del racconto è condensato nel suo incipit in versi, ripreso poi, identico ma in prosa, nel finale del racconto:
...that last summer when life still
appeared to pay heed to individuals, and
when it was easier and more natural to
love than to hate. ${ }^{36}$

La narrazione prende il via con il racconto di un viaggio nell'inverno del i9ı6 alla volta dei monti Urali, dove il protagonista, Serezha, si reca per fare visita alla sorella. Stanco per il viaggio, il protagonista cade in uno stato di sonno-veglia in cui gli tornano alla mente le vicende di «quell'ultima estate in cui la vita pareva ancora prestare attenzione agli individui», l'ultima estate prima della Grande Guerra, trascorsa come tutore presso una nobile famiglia, dove è implicato in complesse vicende amorose. Ma senza addentrarci nei dettagli di una trama che sfugge tra le dita come in genere i racconti dei sogni, ci limitiamo qui a notare come il «tema centrale» del

32 Nell'articolo su Pasternak, accanto al titolo del romanzo, Dottor Zivago, Rosselli indica come anno ed edizione: «(Einaudi, 1964)». Si tratta dell'edizione letta e tuttora presente nella sua biblioteca (Boris Pasternak, Il dottor Zivago, prefazione di E. Montale, Torino, Einaudi, I964, FAR 892) e non della prima edizione assoluta, notoriamente edita da Feltrinelli nel 1957.

33 Rosselli, La fatica di essere autentico, cit., p. I29.

34 Boris Pasternak, The last summer, translated from the Russian by George Reavey; illustrated by Vladimir Konashevich, New York, Avon book, I958, FAR 275. Il racconto uscirà in italiano l'anno successivo: Pasternak, L'ultima estate, traduzione dal russo di Dimitri Erikeev e Adalberto Pace, Milano, Lerici, 1959.

35 Boris Pasternak, Safe conduct: an autobiography and other writings, New York, New Directions, I958, FAR 324 .

36 Pasternak, The last summer, cit., p. 19. 
testo sia, suggerisce Lydia Slater, «la poesia». ${ }^{37}$ Essa è condensata nei ritratti delle donne amate, negli scorci di paesaggio umanizzato che scandiscono la narrazione (un esempio per tutti: «The street-lamps and the mist exchanged beastly yawns») ${ }^{38}$ e nelle pagine in cui il protagonista descrive se stesso nell'atto di scrivere. Forse la parte più intensa del racconto è proprio il momento in cui Serezha inizia ad abbozzare la trama di un'opera teatrale, tratteggiando un racconto nel racconto che è un "puro concentrato di poesia».39 Se sono molti i versi che Pasternak dedica al momento della scrittura - come nel componimento Febbraio scelto da Amelia Rosselli: «Febbraio. Prender l'inchiostro e piangere! / Scrivere di febbraio a singhiozzi» ${ }^{40}$ - in queste pagine l'autore entra nel dettaglio, descrivendo minuziosamente il processo del pensiero teso a raccogliere le parole nonché il gesto della scrittura:

The rain was the first detail in the sketch to stop Serezha. He transferred this detail from an octavo to a quarto-sized sheet, and began to amend and erase in an attempt to arrive at the desired lucidity. In places, he penned words that did not exist in the language. He allowed them to stay temporarily on the paper in the hope that they might, later, guide him through more immediate torrents of rainwater into the sort of colloquial speech, which originated from the intercourse of enthusiasm and usage..$^{41}$

Con un'espressione simile a quella usata da Pasternak («he penned words that did not exist in the language»), Amelia Rosselli descrive non più lo scrittore ma il lettore nell'atto di prendere note a margine dei versi o sulla pagina:

$[\ldots]$

With a slip of the pen you endow it, with thoughts which were never there at all, save that you lurked in the shadows finding out its message. ${ }^{42}$

Rosselli assegna al $t u$, all'alterità, un compito preciso, quello di completare la sua poesia, di «conferirle una beatitudine» ${ }^{43}$ fino a quel momento

37 Cito dall'introduzione di Lydia Slater all'edizione del 1960: Boris Pasternak, The last summer, translated by George Ravey, with an introduction by Lydia Slater, London, Penguin books, 1960, p. II.

38 Pasternak, The last summer, cit., p. 54.

39 Sono, di nuovo, parole di Lydia Slater.

40 Pasternak, Febbraio, in Id., Poesie, cit., p. 3.

4I Pasternak, The last summer, cit., p. 75.

42 Rosselli, Sleep, ora in Ead., L'opera poetica, cit., pp. 982-5.

43 Ibidem. 
assente, arricchendola del suo pensiero anche soltanto con una "svista della penna”. L'atto di scrittura si definisce nei versi di Pasternak e Rosselli in tutta la sua forza liberatrice, in grado di creare pensiero critico e dunque di rendere uguale ogni uomo proprio nella sua diversità e unicità. Non è un caso se il titolo inizialmente pensato da Pasternak per il racconto doveva essere Revoliuttsiia, ovvero Rivoluzione. ${ }^{44}$ Come scrive George Ravey nell'introduzione dell'Ultima estate letta da Rosselli: «In this era of isms and nervous scramble after new aspects of reality and technique, in this competitive cauldron of slogans and apocalyptic expectations, the young poet was slowly moving to a definition of his own independent position».45 Pur nella diversità di tempi e luoghi, Amelia Rosselli condivide con Pasternak un simile orientamento politico. Formatasi sui testi del padre, conservati nella sua biblioteca con sottolineature e note autografe, Amelia Rosselli condivide l'«umanesimo libertario» ${ }^{46}$ da lui delineato, individuando il problema centrale del marxismo - così come di ogni forma di -ismo - «nel ruolo che esso assegna all'elemento umano, al fattore volontà». ${ }^{47}$ Scrive Carlo Rosselli:

\begin{abstract}
Nel sistema marxista abbiamo a che fare con una umanità sui generis, composta di uomini per definizione non liberi, operanti sotto la spinta del bisogno, costretti a ricorrere a metodi produttivi indipendenti dal loro volere e ad accedere a rapporti sociali imperativi. Psicologicamente parlando, l'uomo di Marx non è che l'homo oeconomicus di Bentham. Questa è la sua costante psicologica, allo stesso modo della razza, del clima, ecc. Le reazioni che questo homo oeconomicus offre non sono reazioni spontanee ed autonome, ma determinate dal modificarsi dei rapporti produttivi e quindi dei rapporti sociali..$^{8}$
\end{abstract}

Il punto di incontro fra Pasternak e Rosselli sta proprio nella loro ferma volontà politica di contrapporre la propria voce, le proprie emozioni e i propri sentimenti («Solo i fatti, reprimere i sentimenti / poi ritrovi una "persona" che s'aggancia / fortemente al pavimento») ${ }^{49}$ a ogni forma di repressione della libertà:

44 Cfr. Megan Swift, A Self-conscious Tale: Pasternak's Povest', Canadian Slavonic Papers, December 200o, 42, 4, p. 48I. Sulla genesi del racconto si veda anche Christopher Barnes, Boris Pasternak, 1928-1960. A Literary Biography, Cambridge, Cambridge University Press, I998, vol. II.

45 Cito da George Reavey, introduzione a Boris Pasternak, The last summer, Peter Owen Limited London, 1959, p. I6.

46 Carlo Rosselli, Scritti politici e autobiografici, prefazione di Gaetano Salvemini, Napoli, Polis, I944, p. I6I (FAR 2506: il sintagma "umanesimo libertario" è evidenziato in penna blu da Amelia Rosselli).

47 Carlo Rosselli, Socialismo liberale, Torino, Einaudi, 1973, p. 357.

48 Ibidem.

49 Rosselli, Documento, ora in Ead., L'opera poetica, cit., p. 325. 
Il coraggio di Pasternak è proprio quello di non aver rifiutato di parlare delle emozioni, descrivendole come sorelle alla vita, accompagnatrici accette, descritte senza imbrogli, sfaccettature, nascondigli. E senza vergognarsene.

Oggi amare, provare sentimenti, esprimerli, sembra imbarazzare i poeti. Le emozioni le crediamo doppie. Eppure Pasternak era convinto dell'unicità dei suoi sentimenti, ma certamente aveva frugato a lungo in se stesso per trovare quelli autentici. E se nessuna o quasi nessuna "visione della vita" o programma scaturisce dai suoi versi era forse perché gli sembrò bastare proprio questa fatica.

«Con tutta la mia debolezza giuro di restare con voi» - verso sintomatico; rispecchia la sua modestia e la sua fedeltà, alla poesia come alla rivoluzione. ${ }^{50}$

«Do come see my poetry».51 Pasternak e Rosselli invitano a rimeditare la parola dell'altro e da essa farne scaturire il proprio unico pensiero: «with a slip of the pen you».52 Come l'Amleto del Dottor Zivago, significativamente incluso nella selezione di Amelia Rosselli, occorre combattere il «fariseismo» nella consapevolezza che "vivere una vita non è attraversare un campo»,53 bisogna sforzarsi, «tastare ogni terreno ideologico con inaspettata libertà, singolarità», ${ }^{54}$ esplorare "mille, diverse, a volte opposte verità», ${ }^{55}$ provare $l a$ fatica di essere autentico:<smiles>[AlH2]</smiles>

Lo scritto che in me è folle risponde a tutto questo dolore con parole sempre spero sempre vere.

E vero è il tuo linguaggio: vero il nostro? Vero questo parto indolore? più violento, più triste, più calmo, più dolce d'una tua carezza in fronte.

$[\ldots]^{56}$

sara.sermini@usi.ch

50 Rosselli, La fatica di essere autentico, cit., p. I30.

5I Rosselli, Sleep, ora in Ead., L'opera poetica, cit., p. 982.

52 Ibidem.

53 Cito da Amleto, poesia tratta dal Dottor Zivago, anch'essa scelta da Amelia Rosselli. Cfr. Dieci pagine di Pasternak scelte da Rosselli, cit., p. I.

54 Rosselli, La fatica di essere autentico, cit., p. I30.

55 Ibidem.

56 Rosselli, Documento, ora in Ead., L'opera poetica, cit., p. 328. 